This item was submitted to Loughborough's Research Repository by the author.

Items in Figshare are protected by copyright, with all rights reserved, unless otherwise indicated.

\title{
Equivalent circuit model for coupled complementary metasurfaces
}

PLEASE CITE THE PUBLISHED VERSION

https://doi.org/10.1109/TAP.2018.2860052

PUBLISHER

(C) Institute of Electrical and Electronics Engineers (IEEE)

VERSION

AM (Accepted Manuscript)

LICENCE

CC BY-NC-ND 4.0

REPOSITORY RECORD

Bukhari, Syed, William Whittow, J. C. Vardaxoglou, and Stefano Maci. 2018. "Equivalent Circuit Model for Coupled Complementary Metasurfaces". Loughborough University. https://hdl.handle.net/2134/34339. 
This is the final author version of "Equivalent Circuit Model for Coupled Complementary Metasurfaces" by Bukhari et al., IEEE, Transactions on Antennas \& Propagation, 2018

The paper can be downloaded here:

https://ieeexplore.ieee.org/document/8421284/

DOI: 10.1109/TAP.2018.2860052

Further journal papers by Dr. Whittow can be freely downloaded here: http://publications.Iboro.ac.uk/publications/all/collated/elwgw.html 


\title{
Equivalent Circuit Model for Coupled Complementary Metasurfaces
}

\author{
Syed Sheheryar Bukhari, Will Whittow, Senior Member, IEEE, J(Yiannis) Vardaxoglou, Fellow, IEEE, Stefano \\ Maci, Fellow, IEEE
}

\begin{abstract}
Coupled complementary metasurfaces (CCMTS) exhibit a passband whose frequency is several times lower than that of the individual metasurface (MTS) passband frequency. In this paper we explain this phenomenon and propose a simple and accurate equivalent circuit for CCMTS comprised of slots and their Babinet complement, dipoles. An equivalent circuit is extracted from a coupled EFIE-MFIE equation using a synthetic basis function. The same procedure can be conveniently applied to any CCMTS. The model allows one to estimate the large downshift of resonant frequency and the bandwidth utilizing a simple formula. When used in a subresonant regime, the unit cell may have a dimension of a tenth of a free space wavelength with a moderate value of permittivity between the complementary layers.
\end{abstract}

\section{Index Terms - Metasurfaces, FSS, Equivalent Circuit Model}

\section{INTRODUCTION}

Metamaterials are artificially structured materials constituted by subwavelength locally periodic elements [1]. These elements, also called meta-atoms, can be used to produce exotic electromagnetic properties (like negative refractive index) which are not naturally available [2]. Hence metamaterials can be used to design novel electromagnetic devices which were considered impossible by using naturally occurring materials for example, superlenses with perfect focusing and cloaking devices [3]-[5]. However owing to their $3 \mathrm{~d}$ nature, these materials exhibit strong absorption losses and are also difficult to manufacture, especially at microwave frequencies [6]. Such factors have limited the integration of metamaterials in practical applications. Metasurfaces [7] (thin version of metamaterials) suffer less of these drawbacks. Just like metamaterials their individual building blocks (unit cells) are sub-wavelength in dimensions but due to their planar nature they are significantly easier to manufacture and integrate into nanophotonic and microwave devices [8]. The compact nature of individual unit cells of metasurfaces offers sub-wavelength resolutions and allows them to be characterized through continuous (homogeneized) boundary conditions [9]. This allows metasurfaces to have sub-wavelength spatial variability by selectively modifying the constituent inside the individual unit cells, thus allowing for control and shaping of the wavefronts of the impinging electromagnetic space wave [10][11]. Indeed, the tangential derivative of phase discontinuity across the surface can be modified in such a way to shape the wavefront of the electromagnetic wave transmitted through a metasurface.

The complete arbitrary control of the direction and shape of the wavefronts can be achieved if the metasurface exhibits phase discontinuity ranging from $-\pi$ to $\pi$. This has been achieved using $\mathrm{v}$-shaped nanoantennas and also led to the derivation of the generalised laws of reflection and refraction and the denomination of "phase gradient metasurfaces" [11]. The efficiency of the metasurfaces which offer a complete $2 \pi$ phase control can be improved by using multi-layered metasurfaces as shown in [12]. The phase discontinuity across the metasurface is due to the surface currents excited by the impinging wave on its individual elements. Huygen's equivalence (Schelkunoff's) principle states that these surface currents are equivalent to the tangential electric and magnetic fields, and that they are responsible for the electromagnetic field outside any surface. Therefore, leading to a generalization of phase gradient metasurfaces into the so called Huygen's metasurfaces [13]. These surfaces possess more degrees of freedom in controlling the transmitted field than the phase gradient metasurfaces [11], while having the ability to cancel the reflected field. Although frequency selective surfaces and transmit/reflect-arrays can be employed for achieving a specific electromagnetic response, their individual unit cells are typically not of sub-wavelength dimensions, therefore neither do they offer sub-wavelength spatial resolution nor can they be considered as homogeneous structures [9].

Huygen's metasurfaces can be reflection-less. Among the various possible realizations, these can be formed by using two or more separate sheets of individual planar elements. One sheet introduces an electric field discontinuity while the second sheet has been applied to create a discontinuous magnetic field thereby creating a Huygen's source [13]. The electric and magnetic polarizabilities of these sheets are tailored so that the resulting electric admittance and magnetic impedance are imaginary and equal in order to form elementary Huygen's sources. This result allows perfect transmission without any reflections [14]. The behavior of Huygen's metasurfaces has been explained by the help of an equivalent circuit model. This equivalent circuit model employs a lattice network approach for quantifying the response of a Huygen's metasurface [15]. The lattice network model was based on the approach presented in [16] to explain the anomalous behavior of a dogbone structure employed as a meta-layer for near unity transmission. Similarly equivalent circuit parameters for planar and multi-layered FSSs using a Floquet analysis have been presented in $[17,18]$. A hybrid approach under the assumption that the current is independent of the frequency is employed to extend this circuit modelling to periodic layers with arbitrary shaped scatterers. The initial current distribution is imported from a full-wave analysis and subsequently used to extract the circuit parameters [19]. An equivalent circuit model of fish-net type negative index materials in its closed form has been shown [20]. This model correctly predicts the response for both short and long periods between the adjacent layers. A wideband circuit model for the FSSs surrounded by dielectric materials extracted from integral equations has been presented in [21] and [22]. 
The principle of Huygen's metasurfaces has been extended to optical frequencies as shown in [23] thus making them possible to be used for holography and novel beam generators. Carefully designed Huygen's metasurfaces are capable of increasing the directivity of any source [24]. This allowed the design of a highly directive low profile antenna with an aperture illumination efficiency of near-unity. Although the antenna design resembles to that of Fabry-Perot leaky wave antennas, the replacement of a partial reflective surface with a reflectionless metasurface resulted in overcoming the inherent illumination efficiency limit [25].

Metasurfaces can be also used to control surface waves propagation and radiation [26]. This approach has been adopted to design various metasurface lens antennas [27], [28]. The successful use of metasurfaces to design Luneberg and Maxwell fish-eye antennas has also been demonstrated [29] [31]. Metasurfaces have been successfully shown to be able to control/alter the polarization of an incoming electromagnetic wave [32]. When a periodic modulation of the homogenized impedance is realized along the direction of propagation, the surface wave can be transformed into a leaky wave. Metasurfaces have been used to design leaky-wave antennas [33]. By using anisotropic metasurfaces, special boundary conditions have been imposed on surface waves which convert them to unbounded leaky waves [34]. These metasurface based leaky wave antennas make an excellent candidate for space applications. It has been shown that the polarization, phase and amplitude of the wave radiated from these antennas can also be controlled by imposing of boundary conditions [35]-[37].

The work presented here focusses on explaining the behavior of a different class of metasurfaces inspired from Babinet's Principle. We call them closely coupled complementary metasurfaces. It has been shown in the literature that when two Babinet's complements are placed in close proximity of each other, the resulting structure acts as a band pass structure [38]-[40]. The unit cell size of this structure (at the resonant frequency), as well as the distance between the complementary metalayers, are much smaller with respect to the free space wavelength which allows the structure to be categorized as a metasurface. These types of metasurfaces suffer from time consuming simulations, especially if the scattering matrix approach is adopted. However we propose an analytical, closed form approximation of slot-dipole metasurfaces discontinuity-type boundary conditions which offers a fast and efficient way to analyse and design such a tightly coupled structure. To this end we solve coupled EFIEMFIE integral equations by the use of a single synthetic function. From this formulation, it appears quite clear why combining the two Babinet's complements leads to a downscaling of the resonant frequency. This paper also shows that even though the two Babinet's complements have a natural band pass and band stop response respectively, when they are coupled together to form a single metasurface, the resultant response will always be band pass. The presented structure allows the easy design of Huygen's MTS.

\section{EQUIVALENT MODELS FOR ISOLATED COMPLEMENTARY METASURFACES}

Let us first consider the two geometrically complementary MTSs separately, the first one of slot-type (inductive, or L-type) and the second one of dipole-type (capacitive, or C-type). Both the MTSs are suspended in free space and their metallic parts are lossless. A rectangular reference system $(x, y, z)$ is defined with the $z$ axis normal to the surface and the $x$ and $y$ axes aligned with the axes of the square lattice of the periodic cell. In order to analyze the behavior of the surface when subjected to an incident wave, we exploit the MTS transmission line analogs along the $z$ axis, as shown Fig. 1. These vertical transmission lines are associated with the dominant term in the Floquet-mode (FM) expansion of the electromagnetic field, which is identified as the dominant FM. In the following we will denote $k=\omega \sqrt{\varepsilon_{0} \mu_{0}}$ the free space wavenumber, $\omega$ is the angular frequency, $\varepsilon_{0}$ the free space permittivity and $\mu_{0}$ for the free space permeability and $\zeta=\sqrt{\mu_{0} / \varepsilon_{0}}$ the free-space impedance. Although the discussion that follows is relevant to a general complementary couple, here we will refer to a simple slot (along $x$ ) and dipole (along $y$ ). The slot-type and the dipole type MTS can be represented as a parallel $L_{\mathrm{s}}-C_{\mathrm{s}}$ circuit and a series $L_{\mathrm{d}}-C_{\mathrm{d}}$ circuit, respectively, both in parallel to the transmission line. For the slot type, the inductance $L_{\mathrm{s}}$ determines the dominant behavior at low frequencies, while the capacitance $C_{\mathrm{s}}$ is subdominant at low frequencies. A dual behavior occurs for dipoles, i.e., $C_{\mathrm{d}}$ is dominant and $L_{\mathrm{d}}$ subdominant at low frequencies. The C-type exhibits a band stop response while the L-type has a band pass response around a resonant frequency. For L-type and C-type configurations the $\mathrm{TM}_{\mathrm{z}}$ and $\mathrm{TE}_{\mathrm{z}}$ characteristic impedances of the transmission lines can be written as $Z_{T M}=\zeta \cos \theta_{i}$, and $Z_{T E}=\zeta / \cos \theta_{i}$, respectively, where $\theta_{i}$ is the incidence angle with respect to the normal. For both configurations, a scan in the E-plane $(y z)$ will be $\mathrm{TM}_{\mathrm{z}}$ and a scan in the H-plane will be $\mathrm{TE}_{\mathrm{z}}$.

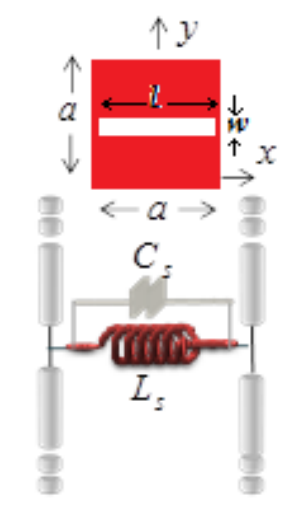

(a)

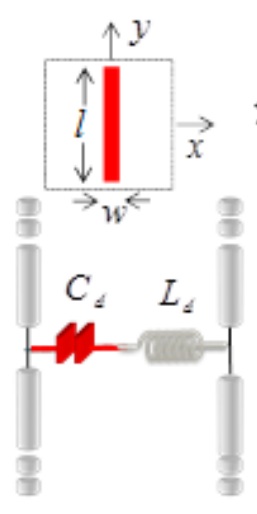

(b)

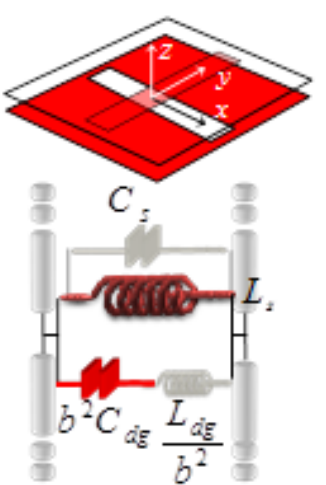

(c)
Figure 1: Equivalent circuit model of MTS. (a) slot in isolation, (b) dipole in isolation, (c) coupled complementary MTS. The distance between the layers is $d$. In all cases the elements are embedded in a periodic environment. Slot and dipole both have length ' $l$ ' and width ' $w$ '.

The homogenized equivalent impedances of the two shunt loads in the equivalent transmission lines have to satisfy the Babinet principle [41], namely 


$$
\left(\frac{\omega L_{s}}{1-\omega^{2} / L_{s} C_{s}}\right)\left(\frac{1-\omega^{2} / L_{d} C_{d}}{\omega C_{d}}\right)=\frac{\zeta^{2}}{4}
$$

Since the right hand side of (1) does not depend on frequency, (1) can be satisfied if and only if

$$
\begin{aligned}
& 1 / \sqrt{L_{d} C_{d}}=1 / \sqrt{L_{s} C_{s}}=\omega_{0} \\
& \sqrt{L_{s} / C_{d}}=\sqrt{L_{d} / C_{s}}=\zeta / 2
\end{aligned}
$$

The above are two independent equations that allows one to find the four components $L_{\mathrm{d}}, C_{\mathrm{d}}, L_{\mathrm{s}}$, and $C_{\mathrm{s}}$ on the basis of the knowledge of the resonant frequency $\omega_{0}$ and one of the four parameters. This is true for any couple of complementary MTS. This is true for any couple of complementary surfaces, provided that the metal is infinitesimally thin and the layer is immersed in free-space. For the particular case of the dipole and slot, the resonance frequency is such that the length is approximately half of the free-space wavelength, like the one of a dipole (slot) in isolation. An accurate estimate of the parameters is obtained by using a single "synthetic" basis function (SBF) in a Method of Moments (MoM) solution of an integral equation. This SBF is the combination of three resonant basis as stated in equation (4).

$$
f_{e}(x, y)=\operatorname{rect}(x, l) \operatorname{rect}(y, w) \sqrt{\frac{2}{l w}} \sum_{n=1}^{3} C_{n} \cos (\pi n x / l)
$$

with $C_{1}=5.88, C_{2}=0.25$ and $C_{3}=0.33$. It is seen that this combination provides excellent results for any phasing. These coefficients are obtained by first solving the integral equations with MoM using three basis functions. The magnitude of each basis of this solution is then used as the co-efficient $C_{n}$. This approach allows synthesizing a single basis function which accurately represents the electric and magnetic currents in the two complementary elements. The SBF for the electric dipole currents is obtained by interchanging $x$ and $y$, that is, $f_{h}(x, y)=f_{e}(y, x)$. The corresponding spectral functions are denoted by $h\left(k_{x}, k_{y}\right)=e\left(k_{y}, k_{x}\right)$ (dimensionally a length). The standard individual EFIE or MFIE integral equations can now be established, and solved by the Galerkin Method of Moments with the SBF. Following the scheme in [38], this leads to the following values for the circuit in Fig.1

$$
\begin{gathered}
L_{d}=\frac{\mu_{0}}{2 a^{2}} \sum_{p q \neq 0,0}\left[\frac{1}{\alpha_{p q}}\right]\left|h_{p q}\right|^{2} ; C_{d}=\frac{\varepsilon_{0} 2 a^{2}}{\sum_{p q \neq 0,0} \alpha_{p q}\left|h_{p q}\right|^{2}} \\
C_{s}=\frac{2 \varepsilon_{0}}{a^{2}} \sum_{p q \neq 0,0} \frac{1}{\alpha_{p q}}\left|e_{p q}\right|^{2} ; L_{s}=\frac{\mu_{0} a^{2}}{\sum_{p q \neq 0,0} 2 \alpha_{p q}\left|e_{p q}\right|^{2}}
\end{gathered}
$$

where $I$ represent the current at the dipole and $V$ the voltage at

where

$$
\alpha_{p q}=\sqrt{\left(k_{x 0}+\frac{p \pi}{a}\right)^{2}+\left(k_{y 0}+\frac{q \pi}{a}\right)^{2}-k^{2}}
$$

$h_{p q}$ and $e_{p q}$ are the basis (testing) spectral functions $h\left(k_{x}, k_{y}\right)=e\left(k_{y}, k_{x}\right)$, evaluated at the wavenumber the slot, while $V_{\mathrm{i}}$ and $I_{\mathrm{i}}$ are the forcing field due to an incident plane wave of TE or TM type, defined as

$k_{x p}=k_{x 0}+\frac{p \pi}{a}, k_{y q}=k_{y 0}+\frac{q \pi}{a}$. The latter may be found in closed form for the SBF in (4). The above expressions should be considered for incidence on the $\mathrm{E}$ plane $(p, q)=(p, 0)$ with TM polarization, and for incidence on the H-plane $(p, q)=(0, q)$ with TE polarization. Correspondingly, $k_{x 0}=k \sin \theta_{i}, k_{y 0}=0, \quad$ for TE-pol/H-plane-incidence, and $k_{x 0}=0, k_{y 0}=k \sin \theta_{i}$ for TM-pol/E-plane-incidence. It can be easily seen that (5) and (6) respects (2) and (3) as expected. Although in (5) and (6) there is dependence of the capacitance and the inductance on the frequency and the incidence, this dependence is very weak and disappears at low frequencies, thus allowing for the identification with the equivalent circuit in Fig. 1.

\section{EQUiVALENT CIRCUIT MODELling OF COUPLED COMPLEMENTARY METASURFACES}

Consider now the MTS constituted of the aforementioned couple, to make closely coupled complementary metasurfaces (CCMTS) placed in the xy-plane, with a small separation distance $d$. The periodicity of the structure is $a$ along both $\mathrm{x}$ - and $\mathrm{y}$-axes. The incoming electromagnetic wave is travelling in the direction of the positive $\mathrm{z}$-axis.

\section{A. Free space between the layers}

For simplicity in the formulation and physical interpretation, first assume that the dielectric material present between the two complimentary Babinet's layers is free space. Later, we provide the formulation for any dielectric material placed in-between. We assume that the thickness of any metal is negligible. A standard EFIE-MFIE couple of integral equations can be established, and solved by the Galerkin Method of Moments, in which a single basis (test) function is used over the slot and the dipole.

By transforming the set of integral equations in the Fourier spectral domain, the following set of algebraic Floquetmode equations can be derived

$$
\begin{array}{ll}
Z_{d} I-b V=V_{i} & \text { spectral EFIE } \\
b I+Y_{s} V=I_{i} & \text { spectral MFIE }
\end{array}
$$

$$
\begin{aligned}
& V_{i}=\left(1-e^{-j \beta_{00} d}\right)\left\{E_{i}^{T E} ; E_{i}^{T M}\right\} \\
& I_{i}=2 e^{-j \beta_{00} d}\left\{\frac{\gamma_{00}}{\zeta k} E_{i}^{T E} ; \frac{k}{\zeta \gamma_{00}} E_{i}^{T M}\right\}
\end{aligned}
$$

where $\beta_{00}=\sqrt{k^{2}-k_{x 0}^{2}-k_{y 0}^{2}}$ is the z-propagation constant of the dominant $(0,0)$ mode, and the first and second term in the brackets are associated with scan in the $\mathrm{H}$ and $\mathrm{E}$ plane, respectively. The elements in (8) are listed below in equation (10). 


$$
\begin{aligned}
& Z_{d}=j \omega L_{d g}+\frac{1}{j \omega C_{d g}} ; Y_{s}=j \omega C_{s}+\frac{1}{j \omega L_{s}} \\
& b=\sum_{p q \neq 0,0} \frac{e^{-\alpha_{p q} d}}{a^{2}} h_{p q} e_{p q}=\sum_{p q \neq 0,0} \frac{e^{-\alpha_{p q} d}}{a^{2}} h_{p q} e_{p q} \\
& L_{d g}=\frac{\mu_{0}}{2 a^{2}} \sum_{p q \neq 0,0}\left[1-e^{-\alpha_{p q} d}\right]\left[\frac{1}{\alpha_{p q}}\right]\left|h_{p q}\right|^{2} \\
& C_{d g}=\frac{\varepsilon_{0} 2 a^{2}}{\sum_{p q \neq 0,0}\left[1-e^{-\alpha_{p q} d}\right] \alpha_{p q}\left|h_{p q}\right|^{2}}
\end{aligned}
$$

where $C_{s}$ and $L_{s}$, as well as $h_{p q}, e_{p q}$ and $\alpha_{p q}$ have the same meaning as in equations (5) and (6) in Section II. The term $Z_{d}$ in EFIE represents the impedance of the periodic dipole MTS in the presence of the ground plane (apertures layer for CCMTS) placed at a distance $d$. As such, the capacitance $C_{d g}$ is much larger than the capacitance $C_{d}$ of the layer in isolation. This intuitive fact is evident through comparison with (5), since $C_{d g}$ contains in the summation the factors $1-e^{-\alpha_{p q} d} \approx \alpha_{p q} d$ which are very small for vanishing $d$. At the same time, the inductance $L_{d g}$ is much smaller than $L_{d}$ for the same reason. The term $b$ is a non-dimensional coupling coefficient that takes into account the radiation of the slots on the dipoles. We observe that the term $Y_{s}$ in (10) is equal to the one in (6), namely the free space admittance of the slot layer in isolation. The term $b$ represents the reciprocal effects i.e. the radiation of the periodic metallic array on the apertures layer. Since the distance between the two complementary layers is extremely small when compared with the resonant frequency wavelength of the metasurface, hence the thickness can be thought of as approaching zero leading to a vanishing voltage driving term in the EFIE (9),

$$
\begin{aligned}
& Z_{d} I-b V=0 \\
& b I+Y_{s} V=I_{i}
\end{aligned}
$$

The solution of (11) yields

$$
\begin{aligned}
& V / I=Z_{d} / b \\
& \frac{V}{I_{i}}=\frac{Z_{d}}{Y_{s} Z_{d}+b^{2}}=\frac{1}{Y_{s}+\left(Z_{d}\right)^{-1} b^{2}}
\end{aligned}
$$

where $I$ is the current flowing in the MTS dipole layer and $V$ is the voltage at the slot MTS, and $I_{\mathrm{i}}$ represents a current generator. Equation (12) shows that the equivalent impedance of two complementary layers is a parallel between the two admittances $Y_{s}$ and $b^{2} Y_{d}$. This leads to the equivalent circuit model as that in Fig. 1c. We notice that all the above formulations can be repeated for any type of shape of slots and dipole, provided there is an analytical form of the SBF.
The equivalent circuit model, gives insight into the behavior of a CCMTS. Keeping in mind that the dominant component at low frequency are $C_{d g}$ and $L_{s}$, The equivalent impedance of the complementary metasurface is a parallel between the inductance of the slot in isolation and the capacitance of the dipole over a ground plane multiplied by the factor $b^{2}$

We observe that an infinitesimal small distance should remain in between the two layers. For instance, if $d$ is exactly equal to zero, then it is similar to the slot being interrupted in the middle by a small piece of metal; looking at (10), $L_{d g}$ and $1 / C_{d g}$ become zero; therefore $Z_{d}$ is zero and the first line of (11) yields $V=0$, in accordance to the fact that the center of the slot is short circuited. Also, the second term in (12) yields $I=I_{i} / b$. This limit approximates the current flowing in the small piece of metal in the middle of the slot. However, it is worth noting that the resonance disappears, along with the fact that the structure is equal to two very small slots on a ground plane, resonating at a frequency which is twice the one of a single isolated slot. The downscaling of the resonant frequency therefore maintains validity when $d$ is extremely small, but finite, meaning that there is an absence of electric contact between dipole and ground-plane.

\section{B. Shift of resonant frequency}

Eq. (12) or the circuit interpretation in Fig. 1c, shows that CCMTS possess a pass-band behavior at a lower frequency compared to the slot layer in isolation. When the dipole layer is placed in close proximity, it causes a resultant increase in the capacitance with respect to the one of the slot in isolation, thus leading to a decrease in the resonant frequency. The shift of the resonant frequency can be estimated by comparing the dominant components of the equivalent networks in Fig. 1 (a) and 1 (c). This leads to the approximate, but simple formula

$$
\frac{f_{s}}{f_{\text {CСMTS }}} \approx \frac{\sqrt{b^{2} C_{d g}+C_{s}}}{\sqrt{C_{s}}}=\sqrt{b^{2} \frac{C_{d g}}{C_{s}}+1}>1
$$

where $C_{s}$ is the capacitance of the slot-MTS in isolation in (6) and $C_{d g}$ is the capacitance of the dipole-MTS at a distance $d$ from a ground plane in (10), in their quasi static limits; $f_{s}$ and $f_{\text {ССMTS }}$ are the respective resonant frequencies of the slot layer in isolation and the CCMTS. Equation (13) effectively represents the miniaturization of the CCMTS compare to the slot layer in isolation. We call this ratio the compression factor of the CCMTS. This formula can approximate any type of coupled MTS, providing we have an estimate of the capacitance of the slot layer in isolation, the dipole layer above a ground plane and of the coupling factor $b$. We note that (13) is valid when a dielectric is placed in between, (provided we use the values of capacitance given in (16) (Section III-D)). The compression factor, as a function of the separation distance between the two layers, for free space, and the practical value of the relative permittivity is shown in Figure 2 (a) and (b) respectively. 


\section{Quality factor and bandwidth}

The shift of frequency implies an increase of the quality factor $Q$ and a reduction of bandwidth. This can be estimated by an approximation similar to the one adopted in (13). For the slot in isolation, $Q$ is, $Q_{s}^{T E} \approx \frac{\zeta}{\cos \theta_{i}} \sqrt{C_{s} / L_{s}}$ for incidence in the H-plane and $Q_{s}^{T M} \approx \zeta \cos \theta_{i} \sqrt{C_{s} / L_{s}}$ for incidence in the $\mathrm{E}$ plane. The $\mathrm{Q}$ factors for the case of CCMTS are $\quad Q_{\text {CCMTS }}^{T E} \approx \frac{\zeta}{\cos \theta_{i}} \sqrt{\frac{C_{s}+b^{2} C_{d g}}{L_{s}}}$ and $Q_{\text {CCMTS }}^{T M} \approx \zeta \cos \theta_{i} \sqrt{\frac{C_{s}+b^{2} C_{d g}}{L_{s}}}$, namely they increase because of the increase of the capacitance. We notice that, in the quasi static limit, the quantities $b, C_{d g}, C_{s}$, are not dependent on $k$ and on the angle $\theta_{i}$; Since $Q^{-1}$ is approximately equal to the $3 \mathrm{~dB}$ bandwidth, the bandwidth at low frequency depends on $\cos \theta_{i}$ and $1 / \cos \theta_{i}$ for the scans in the E-plane and H-plane, respectively. Furthermore,

$$
\frac{Q_{\text {CCMTS }}^{T E, T M}}{Q_{S}^{T E, T M}}=\frac{f_{\text {CCMTS }} \Delta f_{s}}{f_{s} \Delta f_{\text {CCMTS }}} \approx \sqrt{b^{2} \frac{C_{d g}}{C_{s}}+1}
$$

and, by using (13),

$$
\frac{\Delta f_{s}}{\Delta f_{\text {ССMTS }}} \approx b^{2} \frac{C_{d g}}{C_{s}}+1
$$

Namely, due to the coupling with the dipole layer, the bandwidth of the slot layer reduced by approximately the factor equal to the square of the frequency shifting.

\section{Dielectric between the layers}

When a dielectric is present between the two periodic layers, the results are modified as follows:

$$
\begin{aligned}
\mathrm{C}_{\mathrm{dg}} & =\sum_{\mathrm{pq} \neq 0,0}\left(\frac{\mathrm{a}^{2} \varepsilon_{0}\left[\varepsilon_{r} \alpha_{\mathrm{pq}}\left(\mathrm{e}^{2 \alpha_{\mathrm{pq}} \mathrm{d}}+1\right)+\alpha_{\mathrm{pq}}^{\prime}\left(\mathrm{e}^{2 \alpha_{\mathrm{pq}}^{\mathrm{d}}}-1\right)\right]}{\alpha_{\mathrm{pq}}\left(\mathrm{e}^{2 \alpha_{\mathrm{pq}} \mathrm{d}}-1\right) \alpha_{\mathrm{pq}}^{\prime} h_{p q} h^{*}{ }_{p q}}\right. \\
\mathrm{L}_{\mathrm{dg}} & =\sum_{\mathrm{pq} \neq 0,0}\left(\frac{\mu_{0}\left(\mathrm{e}^{2 \alpha^{\prime}{ }_{\mathrm{pq}} \mathrm{d}}-1\right) h_{p q} h^{*}{ }_{p q}}{\mathrm{a}^{2}\left[\alpha_{\mathrm{pq}}^{\prime}\left(\mathrm{e}^{2 \alpha_{\mathrm{pq}} \mathrm{d}}+1\right)+\alpha_{\mathrm{pq}}\left(\mathrm{e}^{2 \alpha_{\mathrm{pq}} \mathrm{d}}-1\right)\right]}\right.
\end{aligned}
$$$$
\mathrm{C}_{\mathrm{s}}=\sum_{\mathrm{pq} \neq 0,0}\left(\frac{\varepsilon_{0}\left[\left(\alpha_{\mathrm{pq}}^{\prime}+\alpha_{\mathrm{pq}} \varepsilon_{r}\right)^{2} \mathrm{e}^{2 \alpha_{\mathrm{pq}} \mathrm{d}}+\left(\alpha_{\mathrm{pq}} \varepsilon_{r}-\alpha_{\mathrm{pq}}^{\prime}\right)^{2}\right] e_{p q} e_{p q}^{*}}{\mathrm{a}^{2}\left[\varepsilon_{r} \alpha_{\mathrm{pq}}\left(\mathrm{e}^{2 \alpha_{\mathrm{pq}} \mathrm{d}}+1\right)+\alpha_{\mathrm{pq}}^{\prime}\left(\mathrm{e}^{2 \alpha_{\mathrm{pq}} \mathrm{d}}-1\right)\right] \alpha_{\mathrm{pq}}^{\prime} \alpha_{\mathrm{pq}}}\right.
$$$$
\mathrm{L}_{\mathrm{s}}=\sum_{\mathrm{pq} \neq 0,0}\left(\frac{\mathrm{a}^{2} \mu_{0}\left[\alpha_{\mathrm{pq}}^{\prime}\left(\mathrm{e}^{2 \alpha^{\prime} \mathrm{pq}^{\mathrm{d}}}+1\right)+\alpha_{\mathrm{pq}}\left(\mathrm{e}^{2 \alpha_{\mathrm{pq}} \mathrm{d}}-1\right)\right]}{\left[\left(\alpha_{\mathrm{pq}}+\alpha_{\mathrm{pq}}^{\prime}\right)^{2} \mathrm{e}^{2 \alpha_{\mathrm{pq}} \mathrm{d}}+\left(\alpha_{\mathrm{pq}}-\alpha_{\mathrm{pq}}^{\prime}\right)^{2}\right] e_{p q} e^{*}{ }_{p q}}\right.
$$$$
\mathrm{b}=\sum_{\mathrm{pq} \neq 0,0}\left(\frac{2 \alpha_{\mathrm{pq}}^{\prime} \mathrm{e}^{\alpha{ }_{\mathrm{pq}} \mathrm{d}} h_{p q} e_{p q}}{\mathrm{a}^{2}\left[\alpha_{\mathrm{pq}}^{\prime}\left(\mathrm{e}^{2 \alpha_{\mathrm{pq}} \mathrm{d}}+1\right)+\alpha_{\mathrm{pq}}\left(\mathrm{e}^{2 \alpha^{\prime} \mathrm{pq}^{\mathrm{d}}}-1\right)\right]}\right.
$$$$
+\frac{2 \alpha_{\mathrm{pq}} \varepsilon_{r} \mathrm{e}^{\alpha^{\prime} \mathrm{pq}^{\mathrm{d}} h_{p q} e_{p q}}}{\mathrm{a}^{2}\left[\alpha_{\mathrm{pq}} \varepsilon_{r}\left(\mathrm{e}^{2 \alpha_{\mathrm{pq}} \mathrm{d}}+1\right)+\alpha_{\mathrm{pq}}^{\prime}\left(\mathrm{e}^{2 \alpha_{\mathrm{pq}} \mathrm{d}}-1\right)\right]}
$$

The approximation in (13) and (15) remains valid for the expressions in (16). The presence of the dielectric permittivity increases the factor $b$ and therefore increases the frequency downshifting. It should be noted that even though the integral equations in (8) and (11) are valid for any periodicity, however the circuit elements' expressions presented in equations (10) and (16) apply only to the structures with equal periodicities in the $\mathrm{x}$ and $\mathrm{y}$-dimensions.

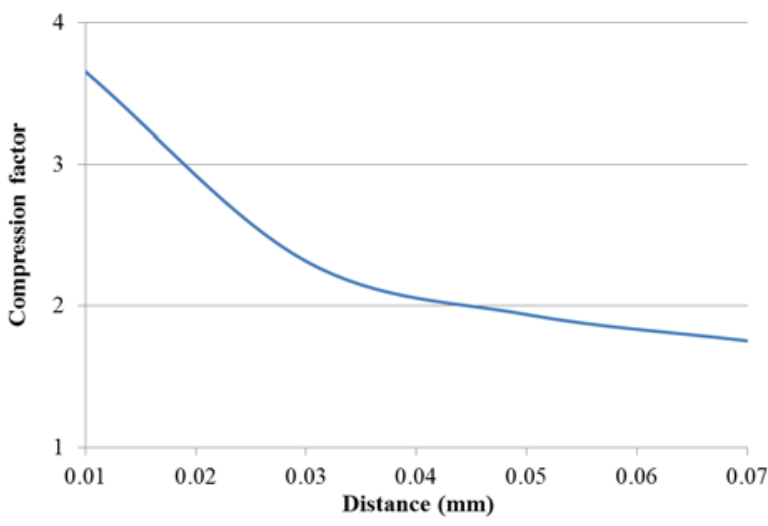

(a)

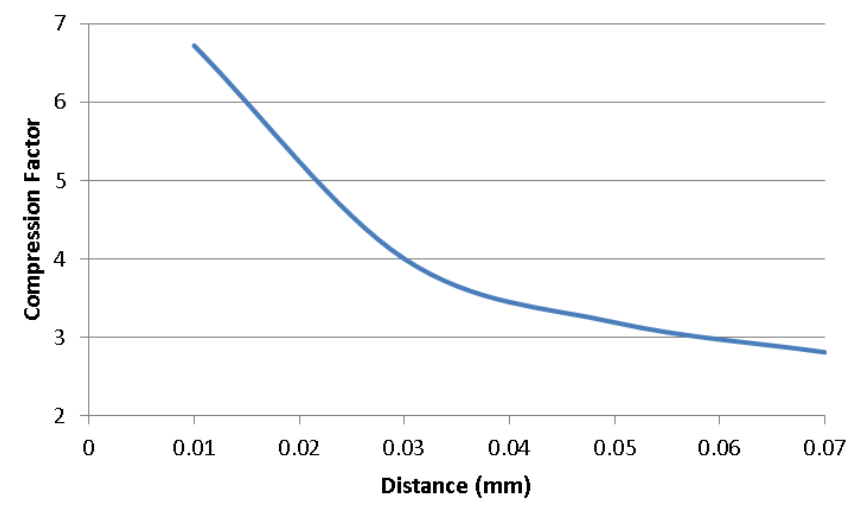

(b)

Figure 2: (a) The compression factor as a function of distance in equation (13) for a CCMTS with free space as the in-between dielectric (for normal incidence). (b) The compression factor as a function of distance in equation (13) for a CCMTS with the in-between dielectric of relative permittivity 3 (This relative permittivity value is equal to that of the material used for experimental verification in Section V). Other parameters are $w=0.3 \mathrm{~mm}, a=$ $10 \mathrm{~mm}, l=9.5 \mathrm{~mm}$. The resonant frequency of a dipole in isolation (with identical parameters) is $15.6 \mathrm{GHz}$.

\section{NUMERICAL TESTS}

Consider first the complementary layers of a dipole and a slot with periodicity of $10 \mathrm{~mm}$ and various lengths, separated by free-space with a separation distance $d=0.07 \mathrm{~mm}$. Both the dipoles and the slots are $w=0.3 \mathrm{~mm}$ wide. The number of Floquet modes used for both layers in order to obtain a convergent solution for the transmission response (and cover the spectra of all the basis functions) was found to be 27 . This structure was then also simulated in CST by using periodic boundary conditions. The slot in isolation for these dimensions exhibits a resonance approximately equal to 15 $\mathrm{GHz}$. The comparison between the transmission response of the equivalent circuit model and the full wave simulations using CST is shown in Figure 3a. A good agreement between 
the phase response of the equivalent circuit model and CST is observed, as demonstrated by Figure 3b.

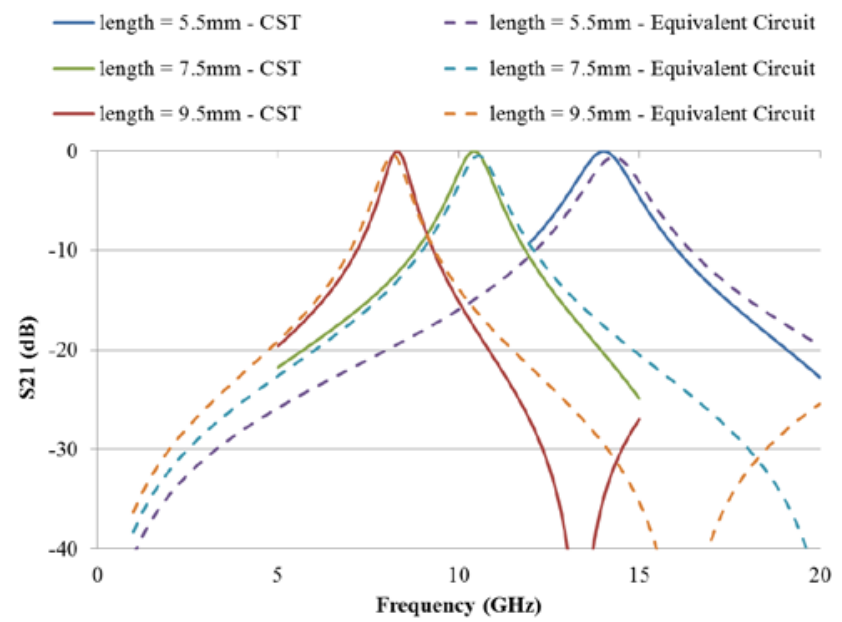

(a)

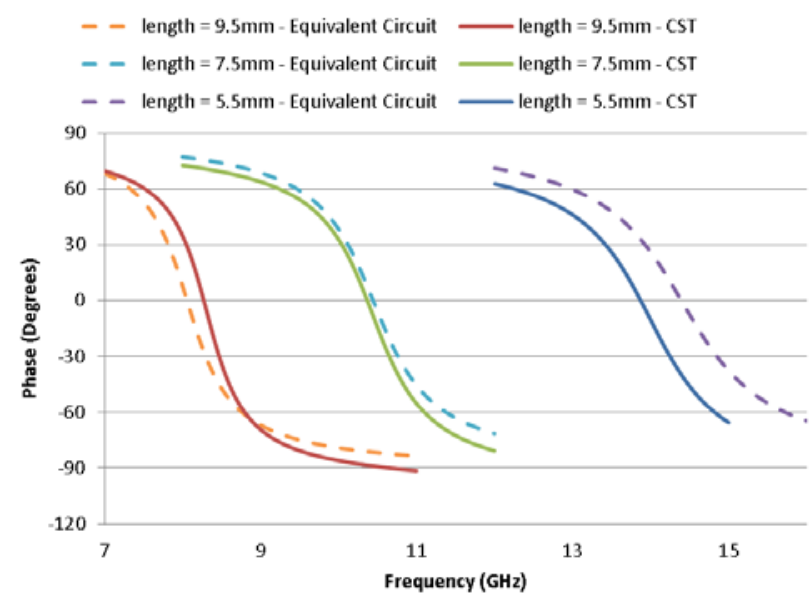

(b)

Figure 3: (a) Transmission response of a dipole-slot metasurface (for norma incidence). (b) Phase response of a dipole-slot metasurface. CST (continuous line) and analytical equivalent circuit model (dashed line). The various colors denote different lengths of dipoles and slots. Other parameters are fixed $(d=$ $0.07 \mathrm{~mm}, w=0.3 \mathrm{~mm}, a=10 \mathrm{~mm}$ )

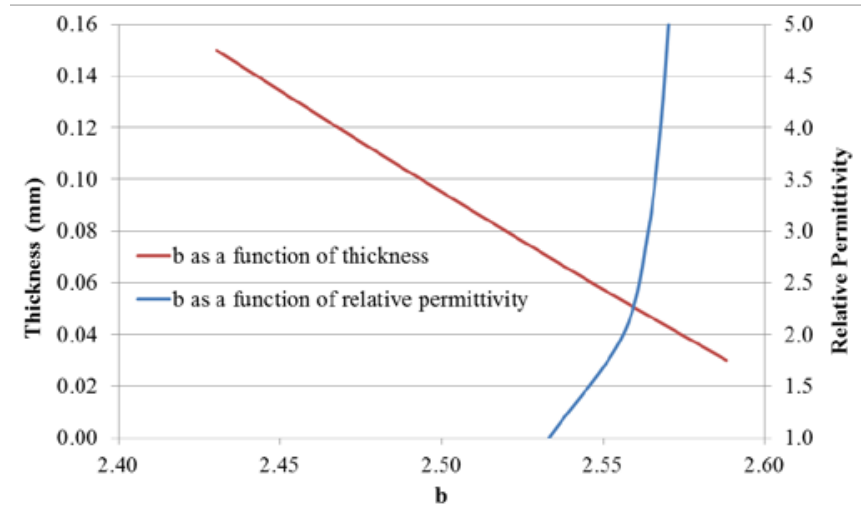

Figure 4: Parameter $b$ as a function of relative permittivity (blue line) for a fixed value of $d(0.07 \mathrm{~mm})$ and $b$ as a function of the thickness $d(\mathrm{~mm})$ for a fixed value of relative permittivity $\left(\varepsilon_{r}=3\right)$ and normal incidence. The other parameters are $w=0.3 \mathrm{~mm}, a=10 \mathrm{~mm}, l=9.5 \mathrm{~mm}$. The resonant frequency of a
CCMTS with relative permittivity $\left(\varepsilon_{r}=3\right)$ and thickness $(d=0.05 \mathrm{~mm})$ is 4.70 $\mathrm{GHz}$.

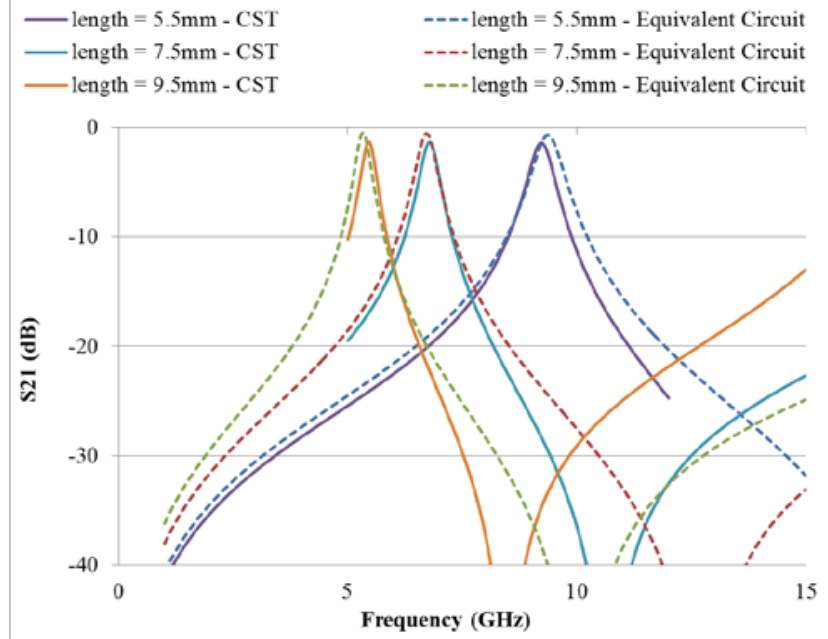

Figure 5: Comparison between the transmission response of a dipole-slot metasurface between the analytical equivalent circuit model for normal incidence and CST simulations with the in-between dielectric having $\varepsilon_{r}=3$. Other parameters are identical to the ones used in Figure 3.

For the length of $9.5 \mathrm{~mm}$ and a dielectric thickness of $0.07 \mathrm{~mm}$, the equivalent circuit model exhibits a resonance at $8.2 \mathrm{GHz}$ while the CST simulations show a resonance at 8.3 $\mathrm{GHz}$. The transmission loss simulated in CST is $0.01 \mathrm{~dB}$ while that observed in the equivalent circuit model is $0.39 \mathrm{~dB}$. Similarly for the length of $5.5 \mathrm{~mm}$ and $7.5 \mathrm{~mm}$, the resonance frequency predicted by the equivalent circuit is $10.5 \mathrm{GHz}$ and 14.3 GHz while the full wave CST simulation exhibit resonances at $10.4 \mathrm{GHz}$ and $14.0 \mathrm{GHz}$. Thus, an excellent agreement between the equivalent circuit model and the full wave simulations has been observed. Figure 4 show the variation of the parameter $b$ with respect to the relative permittivity and the separation distance $d(\mathrm{~mm})$. It is seen that the value of $b$ increases almost linearly with decreasing $d$, and increases for increasing the relative permittivity. Figure 5 shows a successful comparison between CST full-wave results and the circuit model for relative permittivity $\varepsilon_{r}=3$ of the inbetween dielectric. The circuit model uses the parameters in (16). The length of individual elements was changed from $5.5 \mathrm{~mm}$ to $7.5 \mathrm{~mm}$ and $9.5 \mathrm{~mm}$. It can be observed that the resonant frequency of each CCMTS has decreased in comparison to its counterpart in Figure 4. This is because both the capacitance of the dipole adjacent to the ground and the coupling coefficient $b$, increase with increasing $\varepsilon_{r}$ (Fig 3). The shift of frequency and the reduction of bandwidth are in agreement with (13) and (15) respectively.

The effect on the pass-band response to a variation of the thickness of the in-between dielectric is presented in Fig. 6. As the thickness $d$ decreases, the resonant frequency of the CCMTS decreases as well. This can be simply deduced by (13) and Fig. 3. Decreasing $d$ implies increasing the coupling factor $b$ and increasing the capacitance of dipoles which are in close proximity to the ground plane.

The extreme lowering of the resonant frequency also implies a stability of the resonance with respect to the angle of incidence. Figure 7 (a) presents examples of a scan in the $\mathrm{H}$ plane. It is seen, as expected that the resonant frequency is quite stable since $C_{d g}$ and $b$ do not depend on the angle of 
incidence, and the bandwidth increase as $1 / \cos \theta_{i}$ for increasing angle (see Section III-c). Conversely, scanning in the E-plane (shown in Figure 7 (b)) provides decrease of bandwidth like $\cos \theta_{i}$.

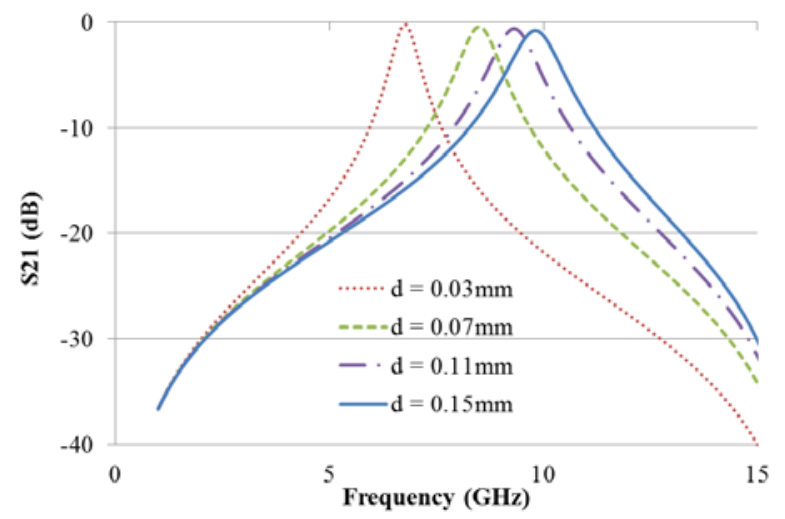

Figure 6: Variation of the resonant frequency with the thickness of the in between dielectric (for normal incidence). The other parameters are $w=$ $0.3 \mathrm{~mm}, a=10 \mathrm{~mm}, l=9.5 \mathrm{~mm}$.

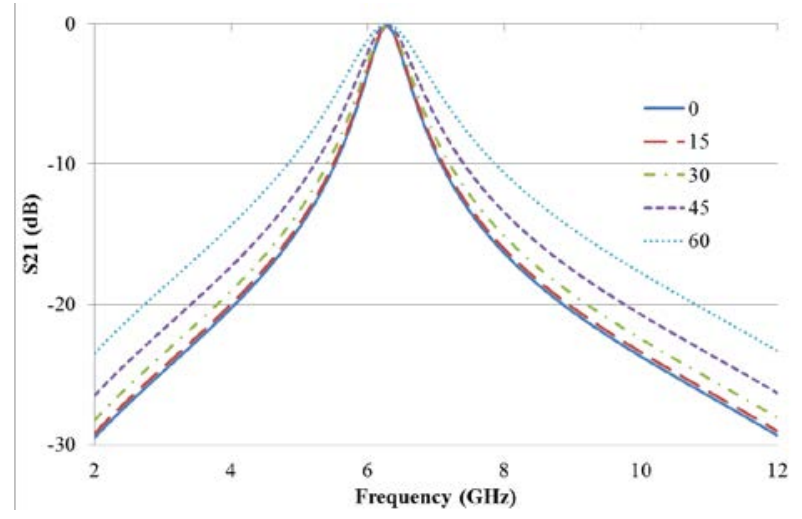

(a)

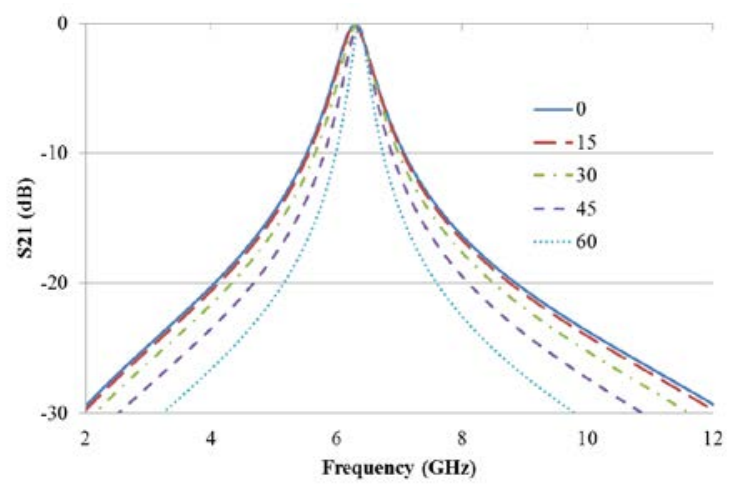

(b)

Figure 7: Effect of the angle of incidence on the passband response of a CCMTS for scanning in (a) H-plane (TE pol) (b) E-plane (TM pol). The other parameters are $w=0.3 \mathrm{~mm}, a=10 \mathrm{~mm}, l=9.5 \mathrm{~mm}, d=0.03 \mathrm{~mm}$ and the inbetween dielectric is free space.

\section{EXPERIMENTAL VERIFICATION}

The equivalent circuit model has also been checked by comparison with experimental results. The fabricated metasurface is shown in Figure 8. The length of the dipoles and apertures was $9.5 \mathrm{~mm}$ with the unit cell periodicity of $10 \mathrm{~mm}$. Each dipole and slot was $0.3 \mathrm{~mm}$ wide. The material used as the in-between dielectric is polyester made by $\mathrm{GTS}^{\mathrm{R}}$. It has a relative permittivity of 3 and a loss tangent of 0.01 . The material was $0.04 \mathrm{~mm}$ thick. The transmission response of this metasurface was measured by placing it between two aligned horn antennas in the Loughborough University anechoic chamber. The two horns were placed at a distance of $3.5 \mathrm{~m}$ and a separator of radar absorbing material was placed between them to ensure that the waves did not propagate around the metasurface. The metasurface was affixed to a $26 \mathrm{~cm} \times 26 \mathrm{~cm}$ aperture in this separator. The total number of unit cells in this metasurface was $676(26 \times 26)$.

The measured transmission response of this metasurface was compared to the simulation results of CST and the equivalent circuit model, see Figure 9. The convergence in this case was achieved with three sinusoidal basis functions and 27 Floquet modes. The comparison between all these results is shown in shown in Figure 9. A satisfactory agreement is observed. The equivalent circuit model for the structure shown in Figure 8 predicts a resonant frequency of $4.45 \mathrm{GHz}$ and an insertion loss of $1.6 \mathrm{~dB}$; the commercial CST software model exhibited a resonance of $4.48 \mathrm{GHz}$ with an insertion loss of $2.1 \mathrm{~dB}$ while the measured transmission response had a resonance of 4.53 $\mathrm{GHz}$ with an insertion loss of $2.3 \mathrm{~dB}$. For convenient quantification when using the GTS material, a rule of thumb is to have $d / \lambda$ approximately equal to $1 / 20$, when a compression factor of 2 is needed in the resonant frequency.

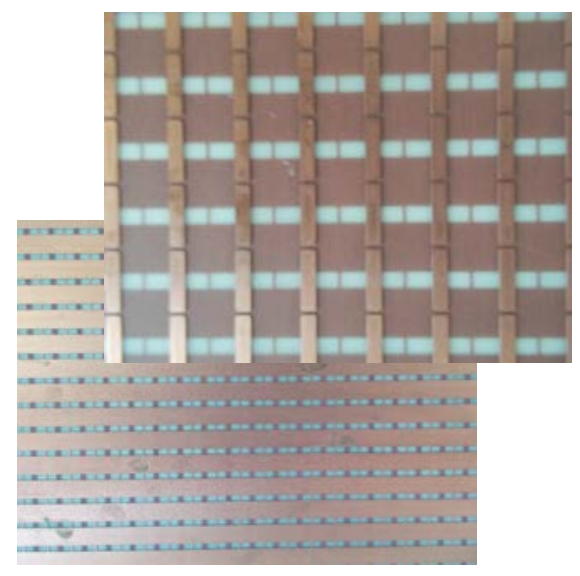

Figure 8: Fabricated Babinet complementary metasurface

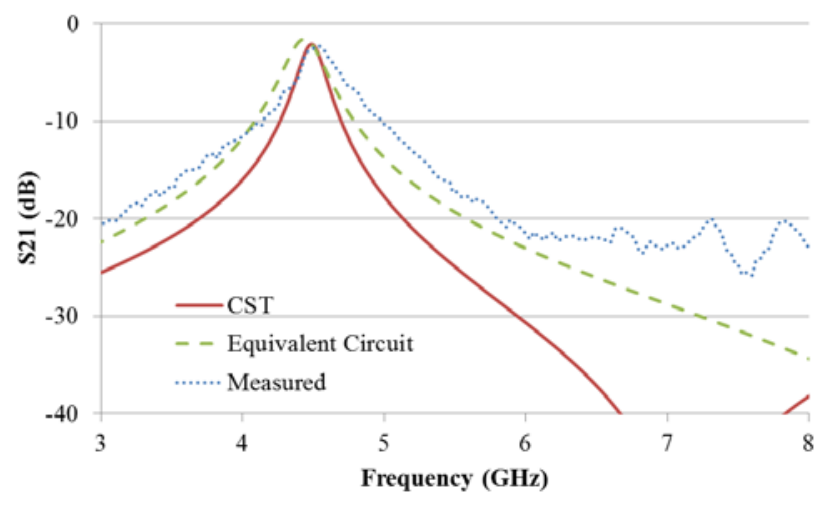

Figure 9: Comparison between the transmission response of a dipole-slot metasurface between the analytical equivalent circuit model, CST simulations and measurements (blue line). 


\section{CONCLUSION}

In this paper we have provided an equivalent analytical model for CCMTS that can explain the basic physics behind the mechanism which is responsible for the frequency shifting, bandwidth, and angular stability. This suggests the potential use of CCMTS for curved surface and phase gradient radomes. We have shown that for vanishing distance between the two coupled layers, the two integral equations for this complementary structure can be reduced to a single magnetic field integral equation. On this basis, an analytical equivalent circuit has been presented, which is simply constituted by the parallel of the inductive admittance of the slots and the capacitive admittance of the dipoles in the presence of a ground plane; the latter divided by the square of a slot/dipole coupling coefficient. Since the response of a parallel $L C$ circuit is always bandpass, such a complementary metasurface will always have a pass-band response. The analytical equivalent circuit also helps explain the compact nature of the elements. The value of the capacitance of the slot layer in isolation is augmented by the capacitance of the dipole layer in close proximity to the slot-ground plane. Since this capacitance element is parallel to the apertures layer, the overall capacitance of the structure increases, leading to a lowering of the resonant frequency and an increase of the $Q$-factor. Comparison between the equivalent circuit model with CST and measured results has demonstrated the accuracy of the simple model. We should finally observe that the effect of coupling between two complementary layers also leads to a lowering of the dispersion equation of the surface wave mode supported by the structure, and that the present formulation can be also adapted to the estimate of this dispersion equation; to this end the formulation in [42] can be used. A size reduction by a factor of ten can be achieved (with the structure presented in this paper) when a material with a relative permittivity of 9.1 and a thickness of $0.06 \mathrm{~mm}$ is used as the in-between dielectric. The manufacture of this kind of material easily falls within the capabilities of current manufacturers. It is finally observed that the present formulation can be used for any couple of CCMTS provided the appropriate basis functions are properly synthesized; as a matter of fact, the significant downshifting of the resonant frequency implies the validity of the LC circuit blocks (in a region around the downshifted resonance, where the elemental cell is small in terms of the wavelength [43]).

\section{REFERENCES}

[1] N. Engheta and R. Ziolkowski, Metamaterials: Physics and Engineering explaorations. Wiley-IEEE Press, 2006.

[2] S. J. Pendry, "Metamaterials and the Control of Electromagnetic Fields," in Conference on Coherence and Quantum Optics, OSA Technical Digest, 2007, p. CMB2.

[3] J. B. Pendry, "Negative Refraction Makes a Perfect Lens," Phys. Rev. Lett., vol. 85, no. 18, pp. 3966-3969, 2000.

[4] N. Engheta, "Ideas for potential applications of metamaterials with negative permittivity and permeability," Advances in Electromagnetics of Complex Media and Metamaterials 2002.

[5] P. Alitalo and S. Tretyakov, "Electromagnetic cloaking with metamaterials,” Mater. Today, vol. 12, no. 3, pp. 22-29, 2009.

[6] C. M. Soukoulis \& M. Wegener, "Past achievements and future challenges in the development of three-dimensional photonic metamaterials,” Nat. Photonics, vol. 5, pp. 523-530,
2011.

[7] C. L. Holloway, E. F. Kuester, J. A. Gordon, J. O’Hara, J. Booth, and D. R. Smith, "An overview of the theory and applications of metasurfaces: The two-dimensional equivalents of metamaterials," IEEE Antennas Propag. Mag., vol. 54, no. 2, pp. 10-35, 2012.

[8] A. M. Urbas, Z. Jacob, L. Dal, H. Rubinsztein-dunlop, A. Forbes, M. V Berry, H. Chen, A. J. Taylor, and N. Yu, “A review of metasurfaces : physics and applications,” Reports on Progress in Physics, Volume 79, Number 7, 2016.

[9] S. B. Glybovski, S. A. Tretyakov, P. A. Belov, Y. S. Kivshar, and C. R. Simovski, "Metasurfaces : From microwaves to visible,” Phys. Rep., vol. 634, pp. 1-72, 2016.

[10] N. Yu and F. Capasso, "Flat optics with designer metasurfaces,” Nat. Mater., vol. 13, no. January, pp. 139150, 2014.

[11] F. Capasso and Z. Gaburro, "Light Propagation with Phase Reflection and Refraction,” Science (80)., vol. 334, pp. 333337, 2011.

[12] F. Monticone, N. M. Estakhri, and A. Alu, "Full Control of Nanoscale Optical Transmission with a Composite Metascreen,” Phys. Rev. Lett., vol. 203903, no. May, pp. 1-5, 2013.

[13] M. Selvanayagam and G. V Eleftheriades, "Discontinuous electromagnetic fields using orthogonal electric and magnetic currents for wavefront Manipulation," Optics Express, vol. 21, no. 12, pp. 3159-3165, 2013.

[14] C. Pfeiffer and A. Grbic, "Metamaterial Huygens' Surfaces: Tailoring Wave Fronts with Reflectionless Sheets," Phys. Rev. Lett., vol. 197401, no. May, pp. 1-5, 2013.

[15] M. Selvanayagam and G. V. Eleftheriades, "Circuit modeling of Huygens surfaces,” IEEE Antennas Wirel. Propag. Lett., vol. 12, no. 2, pp. 1642-1645, 2013.

[16] F. Capolino, A. Vallecchi and M. Albani, "Equivalent Transmission Line Model With a Lumped X-Circuit for a Metalayer Made of Pairs of Planar Conductors," IEEE Trans. Antennas Propag., vol. 61, no. 2, pp. 852-861, 2013.

[17] R. Dubrovka, J. Vazquez, C. Parini, and D. Moore, "Equivalent circuit method for analysis and synthesis of frequency selective surfaces," IEE Proc. - Microwaves, Antennas Propag., vol. 153, no. 3, pp. 213-220, 2006.

[18] D. M. R Dubrovka, J Vazquez, C Parini, "Multi-frequency and multi-layer frequency selective surface analysis using modal decomposition equivalent circuit method," IET Microwaves, Antennas Propag., vol. 3, no. April 2008, pp. 492-500, 2009.

[19] F. Mesa, M. García-Vigueras, F. Medina, R. Rodríguezberral, and J. R. Mosig, "Circuit-Model Analysis of Frequency Selective Surfaces With Scatterers of Arbitrary Geometry,” IEEE Antennas Wirel. Propag. Lett., vol. 14, pp. 135-138, 2015.

[20] V. Torres, F. Mesa, M. Navarro-cía, R. Rodríguez-Berral, M. Beruete, and F. Medina, “Accurate Circuit Modeling of Fishnet Structures for Negative-Index-Medium Applications," IEEE Trans. Microw. Theory Tech., vol. 64, no. 1, pp. 15-26, 2016.

[21] F. Mesa, R. Rodríguez-Berral, M. García-vigueras, F. Medina, and J. R. Mosig, "Simplified Modal Expansion to Analyze Frequency-Selective Surfaces : An Equivalent Circuit Approach,” IEEE Trans. Antennas Propag., vol. 64, no. 3, pp. 1106-1111, 2016.

[22] R. Rodríguez-Berral, F. Mesa, and F. Medina, “Analytical Multimodal Network Approach for 2-D Arrays of Planar Patches / Apertures Embedded in a Layered Medium," IEEE Trans. Antennas Propag., vol. 63, no. 5, pp. 1969-1984, 2015.

[23] C. Pfeiffer, N. K. Emani, A. M. Shaltout, A. Boltasseva, V. M. Shalaev, and A. Grbic, "Efficient Light Bending with Isotropic Metamaterial Huygens’ Surfaces,” Nano Lett., vol. 
14, pp. 2491-2497, 2014.

[24] A. Epstein and G. V Eleftheriades, "Passive Lossless Huygens Metasurfaces for Conversion of Arbitrary Source Field to Directive Radiation,” IEEE Trans. Antennas Propag., vol. 62, no. 11, pp. 5680-5695, 2014.

[25] A. Epstein, J. P. S. Wong, and G. V Eleftheriades, “Cavityexcited Huygens' metasurface antennas for near-unity aperture illumination efficiency from arbitrarily large apertures," Nat. Commun., vol. 7, pp. 1-10, 2016.

[26] E. Martini, M. Mencagli, D. González-Ovejero, and S. Maci, "Flat Optics for Surface Waves," IEEE Trans. Antennas Propag., vol. 64, no. 1, pp. 155-166, 2016.

[27] Haipeng Li, GuangmingWang, Jiangang Liang, Xiangjun Gao, Haisheng Hou, and Xinyan Jia, "Single-Layer Focusing GradientMetasurface for Ultrathin Planar Lens Antenna Application,” IEEE Trans. Antennas Propag., vol. 65, no. 3, pp. 1452-1457, 2017.

[28] C. Pfeiffer and A. Grbic, "Planar Lens Antennas of Subwavelength Thickness: Collimating Leaky-Waves With Metasurfaces," IEEE Trans. Antennas Propag., vol. 63, no. 7, pp. 3248-3253, 2015.

[29] Y. Shi, K. Li, J. Wang, L. Li, and C. Liang, “An Etched Planar Metasurface Half Maxwell Fish-Eye Lens Antenna," IEEE Trans. Antennas Propag., vol. 63, no. 8, pp. 37423747, 2015.

[30] M. Bosiljevac, M. Casaletti, F. Caminita, Z. Sipus, and S. Maci, "Non-Uniform Metasurface Luneburg Lens Antenna Design,” IEEE Trans. Antennas Propag., vol. 60, no. 9, pp. 4065-4073, 2012.

[31] S. Maci, G. Minatti, M. Casaletti, and M. Bosiljevac, "Metasurfing: Addressing Waves on Impenetrable Metasurfaces," IEEE Antennas Wirel. Propag. Lett., vol. 10, pp. 1499-1502, 2012.

[32] M. Selvanayagam and G. V Eleftheriades, "Polarization Control Using Tensor Huygens Surfaces,” IEEE Trans. Antennas Propag., vol. 62, no. 12, pp. 6155-6168, 2014.

[33] D. Blanco, E. Rajo-Iglesias, S. Maci, and N. Llombart, "Directivity Enhancement and Spurious Radiation Suppression in Leaky-Wave Antennas using Inductive Grid Metasurfaces,” IEEE Trans. Antennas Propag., vol. 63, no. 3, pp. 891-900, 2015.

[34] G. Minatti, S. Maci, P. De Vita, A. Freni, and S. Member, “A Circularly-Polarized Iso fl ux Antenna Based on Anisotropic Metasurface," IEEE Trans. Antennas Propag., vol. 60, no. 11, pp. 4998-5009, 2012.

[35] G. Minatti, M. Faenzi, E. Martini, F. Caminita, P. De Vita, D. González-Ovejero, M. Sabbadini, and S. Maci, "Modulated Metasurface Antennas for Space: Synthesis, Analysis and Realizations,” IEEE Trans. Antennas Propag., vol. 63, no. 4, pp. 1288-1300, 2015.

[36] A. T. Pereda, F. Caminita, E. Martini, I. Ederra, J. C. Iriarte, R. Gonzalo, and S. Maci, "Dual Circularly Polarized Broadside Beam Metasurface Antenna," IEEE Trans. Antennas Propag., vol. 64, no. 7, pp. 2944-2953, 2016.

[37] G. Minatti, F. Caminita, E. Martini, M. Sabbadini, and S. Maci, "Synthesis of Modulated-Metasurface Antennas With Amplitude , Phase , and Polarization Control," IEEE Trans. Antennas Propag., vol. 64, no. 9, pp. 3907-3919, 2016.

[38] D. S. Lockyer, J. C. Vardaxoglou, and R. A. Simpkin, “Complementary Frequency Selective Surfaces,” IEE Proc. Microwaves, Antennas Propag., vol. 147, no. 6, pp. 501-507, 2000.

[39] S. S. Bukhari, W. G. Whittow, J. C. Vardaxoglou, and S. Maci, "Dipole-slot-dipole metasurfaces," IET Microwaves, Antennas Propag., vol. 10, no. Lapc 2015, pp. 1384-1389, 2016.

[40] S. S. Bukhari, W. G. Whittow, and S. Maci, "Square Loop Complementary Frequency Selective Surfaces,” in 2015 IEEE International Symposium on Antennas and Propagation
\& USNC/URSI National Radio Science Meeting, 2015, pp. 1258-1259.

[41] H. G. Booker, "Slot Aerials and Their Relation to Complementary Wire Aerials (Babinet’s Principle),” J. Inst. Electr. Eng. - Part IIIA Radiolocation, vol. 93, no. 4, pp. 620-626, 1946.

[42] D. Gonzalez-Overjero, E. Martini, S. Maci, "Surface waves supported by metasurfaces with self complementary geometries,” IEEE Trans. Antennas Propag., vol. 63, n. 9, pp. 3982, 3993, Sept. 2015.

[43] M. Mencagli Jr., E. Martini, S. Maci, "Surface wave dispersion for anisotropic metasurfaces constituted by elliptical patches” IEEE Trans. Antennas Propag., vol. 63, no. 7, pp. 2992 - 3003, July 2015.

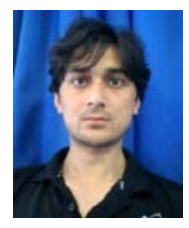

Syed Sheheryar Bukhari completed his Ph.D. in Electronics and Electrical Engineering (2017) from Loughborough University, U.K.. Currently, he is working as a Research Associate in the Wireless Communications Research Group (WiCR) in the Wolfson School of Mechanical, Electrical and Manufacturing Engineering at Loughborough University, U.K..

His research interests include periodic structures, metasurfaces, computational electromagnetics, artificial dielectrics and additive manufacturing.

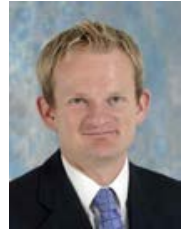

William G. Whittow (M'12-SM'12) received the B.Sc. degree in physics and the Ph.D. degree in computational electromagnetics from the University of Sheffield, Sheffield, U.K., in 2000 and 2004, respectively. From 2004 to 2012, he was a Research Associate at Loughborough University, Loughborough, U.K. In 2012, he became a Lecturer in Electronic Materials Integration, University of Loughborough. He became a Senior Lecturer in 2014 and a reader in Radio Frequency Materials in 2018, in the Wolfson School of Mechanical, Electrical and Manufacturing Engineering at Loughborough University. He has authored more than 180 peer-reviewed journal and conference papers in topics related to electromagnetic materials, synthetic dielectrics, dielectric measurements, 3D-printing, wearable antennas, VHF antennas, specific absorption rate, FDTD, specific absorption rate, metamaterials, heterogeneous substrates, embroidered antennas, inkjet printing, electromagnetic compatibility, RFID tags, phantoms and genetic algorithms. From 2007-2011, Dr. Whittow was the Coordinating Chair of the Loughborough Antennas and Propagation Conference (LAPC). In 2017, he won the Women in Engineering Men As Allies Award. He is an Associate Editor of Electronics Letters. He serves on the technical programme committees of several IEEE international conferences. He has been asked to give 13 invited conference presentations; a 4-day invited workshop on bioelectromagnetics and teach about dielectric measurements at the European School of Antennas. His academic journal papers can be freely downloaded here: http://publications.lboro.ac.uk/publications/all/collated/elwgw.html

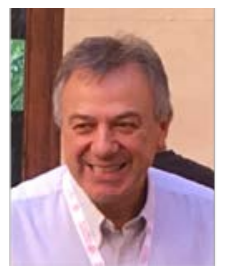

Professor J. (Yiannis) C. Vardaxoglou (F'12) completed his B.Sc. in mathematical physics (1982) and his Ph.D. in Electronics (1985) at the University of Kent, UK. He joined Loughborough University as a lecturer in 1988 and was promoted to Senior Lecturer in 1992 and Professor or Wireless Communications in 1998. He served as the Dean of the School of Electronic, Electrical and Systems Engineering at Loughborough University from 2011-2012.He established the 30 year old Wireless Communications Research (WiCR) group at Loughborough University and founded the Centre for Mobile Communications Research (CMCR). He is the director of Symeta research centre,(www.symeta.co.uk) funded by an EPSRC Grand Challenge award, researching in a wide-ranging topics applicable to cutting-edge wireless communications technology. His current research focuses primarily on metamaterial structures, additive manufacturing (3D printing) for $\mathrm{RF} / \mathrm{micro} / \mathrm{mm}$ wave engineering. Symeta collaborates with many internationally leading companies and universities. He was recently awarded a prestigious EPSRC's Grand Challenge £5M (FEC) award: Synthesizing 3D Metamaterials for RF, Microwave and $\mathrm{THz}$ Applications, (http://gow.epsrc.ac.uk/NGBOViewGrant.aspx?GrantRef=EP/N010493/1). 
He has served as a consultant to various industries, holds six patents and is the Technical Director of Antrum Ltd. He has attracted research funding from industry and has been awarded 18 EPSRC research grants. He has published over 300 refereed journals and conference proceeding papers (with 6500 citations) and has written a book on FSS. He was Chairman of the Executive Committee of the IET's Antennas and Propagation Professional Network in the UK and chaired the IEEE's distinguish lecturer program of the Antennas and Propagation Society (APS) for five years. He founded the Loughborough Antennas \& Propagation Conference (LAPC), which has been running since 2005. He has chaired numerous IEE//IET events and has served on the Steering Committee of the European Conference on Antennas and Propagation, EuCAP. He was the General Chair of EuCAP '07. He was elected a Fellow of the Royal Academy of Engineers in 2011 and a Fellow of the Institute of Electrical and Electronics Engineers in 2012.

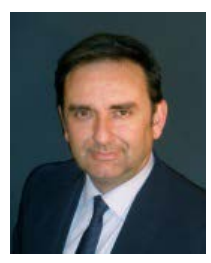

Stefano Maci received the Laurea (cum laude) degree from the University of Florence, Florence, Italy, in 1987. Since 1997, he has been a Professor with the University of Siena, Siena, Italy. His current research interest includes high-frequency and beam representation methods, computational electromagnetics, large phased arrays, planar antennas, reflector antennas and feeds, metamaterials, and metasurfaces. His research activity is documented in 140 papers published in international journals, (with 90 in IEEE journals), ten book chapters, and about 300 papers in international proceedings. These papers have received around 4900 citations. Mr. Maci has been a member of the Technical Advisory Board of 11 international conferences, a member of the Review Board of six International Journals since 2000. He organized 25 special sessions in international conferences, and he held ten short courses in the IEEE Antennas and Propagation Society (AP-S) Symposia about metamaterials, antennas and computational electromagnetics. From 2004 to 2007, he was a WP Leader of the Antenna Center of Excellence (ACE, FP6 EU) and from 2007 to 2010, he was an International Coordinator of a 24institution consortium of a Marie Curie Action (FP6). Since 2010, he has been a Principal Investigator of six cooperative projects financed by the European Space Agency. In 2004, he founded the European School of Antennas, a post graduate school that currently comprises 30 courses on antennas, propagation, electromagnetic theory, and computational electromagnetics, with 150 teachers from 15 countries. He was Co-Founder of two spin-off companies. He was a former member of the AdCom of AP-S, an Associate Editor of the IEEE Transactions on Antennas and Propagation, the Chair of the Award Committee of IEEE AP-S, and a member of the Board of Directors of the European Association on Antennas and Propagation (EurAAP). He was the Director of the Ph.D. Program in information engineering and mathematics of the University of Siena from 2008 to 2015, and also a member of the National Italian Committee for Qualification to Professor from 2013 to 2015. He was a former member of the Antennas and Propagation Executive Board, Institution of Engineering and Technology, U.K. He is also the Director of the Consortium FORESEEN, currently involving 40 European institutions. He is presently international coordinator of the EU project Nanoarchitectronics (H2020 FET Open CSA). He was a Distinguished Lecturer of the IEEE AP-S from 2010 to 2014, and the recipient of the EurAAP Award in 2014, of the IEEE Schelkunoff Transaction Prize 2016, and the Chen-To Tai Distinguished Educator Award 2016. 